\title{
Chondrosarcoma of the Scapula in a Patient with Maffucci Syndrome - Case Report and Literature Review \\ F Birsasteanu ${ }^{1,2}$, D Costachescu ${ }^{2}$
}

\begin{abstract}
Maffucci syndrome (MS) is a very rare, non-hereditary dysplasia, manifested by multiple enchondromas and haemangiomas. Malignant transformation of these lesions is seen in up to $33 \%$ of the cases. There is also a very rare association with secondary musculoskeletal deformaties. We present a case of a patient with Maffucci syndrome and an associated chondrosarcoma of the scapula. Treatment consisted of surgical resection. Because of the low grade of the tumor (G1), additional treatment radiotherapy/chemotherapy), was not necessary. Maffucci syndrome is an extremely rare mesodermal dysplasia. Malignant transformation of the associated enchondromas is common (33\%) and should be considered whenever a change of the clinical course occurs. Clinical, as well as imaging follow-up (MRI) is required because of the relatively high rate of malignant degeneration of the skeletal lesions. However, random, X-ray examinations give little additional information on malignant transformation and are considered useless.
\end{abstract}

Keywords: Chondrosarcoma, enchondroma, hemangioma, maffucci syndrome

From: ${ }^{1}$ University of Medicine and Pharmacy "Victor Babes" Timisoara, Romania, ${ }^{2}$ Department of Radiology, County Hostpital Timisoara, Romania.

Correspondence: Dr D Costachescu, Department of Radiology, Country Hospital, Timisoara, Romania. E-mail: costachescu@yahoo.com 


\section{INTRODUCTION}

Maffucci syndrome (MS) is an extremely rare entity characterized by a combined occurrence of multiple enchondromas and venous malformations such as haemangiomas(1) Unlike other mesenchmal dysplasias like Ollier's disease (OD), the lesions of MS have more of a tendency to malignant transformation(2), which indeed is recognized as a principal factor affecting prognosis. The various possible neoplasms that may occur are musculoskeletal malignancy, gliomas, gastrointestinal adenocarcinoma, pancreatic carcinomas and ovarian tumours.

\section{CASE REPORT}

A 46-year old male, presented at the Emergency Unit of Timisoara County Hospital with pain, swelling and functional loss of his left shoulder. A radiograph of that region showed a large lytic mass with a condromatous matrix involving the left scapula (Fig.1).

After the radiograph, the patient underwent a CT scan, MRI scan (Fig. 2) as well as a PET-CT, all favouring the diagnosis of a chondrosarcoma.

Imaging findings vary according to the different subtype of chondrosarcoma, but the general features are the same. Usually, chondrosarcomas are large masses when they are diagnosed, over $4 \mathrm{~cm}$ in diameter in general and over $10 \mathrm{~cm}$ in $50 \%$ of cases (2).

Further clinical examinations showed bony painless swellings on the metacarpal regions as well as phalanges of his right and left hands which proved to be enchondromas as well as subcutaneous haemangiomas. The presence of multiple enchondromas associated with soft tissue haemangiomas are the hallmark features of Maffucci syndrome. 
The needle biopsy of the scapular lesion confirmed a low-grade chondrosarcoma (G1). A total scapular resection was performed (Tichoff-Lindberg procedure) with the preservation of the humerus. No additional radiotherapy or chemotherapy was performed.

\section{DISCUSSION}

The imaging appearance of chondrosarcomas varies according to the different types, but the general features are the same. It is not difficult to differentiate a high grade chondrosarcoma from an enchondroma, but it is almost impossible to differentiate a low grade chondrosarcoma from an enchondroma. Recent studies(3) showed the importance of dynamic contrast MRI in the differentiation of low grade chondrosarcomas from benign cartilaginous tumours. However, standard MRI remains a complementary method for the one mentioned above.

Some of the general imaging features of chondrosarcomas include:

On plain $x$-ray and computed topography: the lesions are lytic, present with intralesional calcifications(“popcorn calcifications"), endosteal scalloping (it the cortex is affected more than $2 / 3$, this is a sign of malignancy(3)). There can be seen a "moth eaten appearance" in high grade lesions like myxoid, mesenchymal and undifferentiated chondrosarcomas.

On bone scan: chondrosarcomas usually demonstrate avid uptake on bone scan, seen in over $80 \%$ of cases(3). This is useful in some degree in helping to distinguish a low grade chondrosarcoma from an enchondroma (increased uptake in only $21 \%$ of cases).

On magnetic resonance imaging the following is observed: T1 - low to intermediate signal; T2: high intensity in noncalcified areas ; gradient echo/SWI: blooming of calcified portions; T1 C+ most demonstrate heterogeneous moderate to intense contrast enhancement; the enhancement 
can be septal or peripheral rim-like which corresponds to a fibrovascular septation that is seen between lobules of hyaline cartilage.

Maffucci syndrome, also known as dyschondro-dysplasia with haemangiomas, enchondromatosis with multiple cavernous haemangiomas, Kast syndrome, haemangiomatosis chondrodys-trophica, enchondromatosis Spranger type II, was first described by Maffucci in 1881 although possible cases have been known to exist since 1835 . It can occur in all races and has no gender predilection $(4,5)$. It is characterized by multiple enchondromas, haemangiomas and, less often, lymphangiomas in $25 \%$ of cases(6).

Maffucci's syndrome is clinically similar to and thus sometimes confused with Ollier's disease because the skeletal lesions in both these types of enchondromatosis are the same. However, Ollier's disease has no haemangiomas.

The skeletal and soft tissue lesions (vascular lesions) of the limbs are usually asymmetrically distributed, with unilateral changes seen in about $50 \%$ of the cases $(1,7)$. The haemangiomas in Maffucci syndrome are located in the subcutaneous soft tissues and appear as bluish subcutaneous nodules, which can be emptied by means of manual compression. However, they can be found anywhere in the body, not only in the subcutaneous tissues as involvement of the viscerae and mucous membranes by haemangiomas has also been reported in different papers $(1,4)$.

The most frequent sites of enchondromas are the metacarpals and phalanges of the hands. The feet are less commonly affected (7). Clinical manifestation of the skeletal lesions can be a painless swelling of a digit, a pathological fracture or no manifestation at all.

One of the most feared complications of Maffucci Syndrome is the malignant transformation of the lesions. Reviewing of the literature shows a big discrepancy in the rate of 
malignant transformation of the skeletal lesions in Maffucci's syndrome with a varying overall rate of $23 \%-100 \%$ as shown in different studies $(4,7,8)$. This is in contrast to a single enchondroma which rarely becomes malignant. Some studies (9) reported sarcomatous transformation of an enchondroma in only $15.2 \%$ of the cases. In a ten year follow up by Biber et al, sarcomatous transformation occured in $25 \%$ of the cases of enchondromas, whereas Sun et al reported a prevalence of malignant transformation in $56 \%$ of the cases.

In the absence of clinical symptoms and no worrying imaging findings, no treatment is required $(5,7)$. Surgery is indicated only in cases which present with complications such as pathological fractures, malignant transformation and growth defects $(5,7)$. The aim of surgery is to remove the tumour with safety margins. After the surgery is complete, a histological diagnosis is required. In the index case surgery was the only treatment required. The patient is followed up clinically and by regular MRIs every six months.

\section{CONCLUSION}

Maffucci syndrome is an exceedingly rare mesodermal dysplasia. Malignant transformation of the associated enchondromas is common, and should be considered whenever a change of the clinical course occurs. If complete surgical resection can be achieved, no additional treatment like radiotherapy or chemotherapy is necessary. The prognosis of these chondrosarcomas is quite favourable.

\section{AUTHORS' NOTE}

The authors report no conflict of interest and no funding was received on this work. 


\section{REFERENCES}

1. Amary MF DS, Halai D, Eskandarpour M, Berisha F, Bonar F et al. Ollier disease and Maffucci syndrome are caused by somatic mosaic mutations of IDH1 and IDH2. Nat Genet. 2011; 43: 1272-5.

2. Verdegaal SHM BJ, Pansuriya TC, Grimer RJ, Ozger H, Jutte PC, San Julian M. Incidence, predictive factors, and prognosis of chondrosarcoma in patients with Ollier disease and Maffucci syndrome: an international multicenter study of 161 patients. Oncologist. 2011; 16: 1771-9.

3. De Coninck T JL, Sys G, Huysse W, Verstraeten T, Forsyth R et al. Dynamic contrastenhanced MR imaging for differentiation between enchondroma and chondrosarcoma. Eur Radiol. 2013; 23: 3140-52.

4. D’Angelo L ML, Narducci A, et al. Ollier disease. Childs Nerv Syst 2009; 25: 647.

5. Pannier S L-ML. Hereditary multiple exostoses and enchondromatosis. Best Pract Res Clin Rheumatol 2008; 22: 45-54.

6. Karacalioglu O. Combined Tc-99m MDP and Tc-99m Labeled RBC Imaging in a Case f Maffucci's Syndrome. Clin Nuclear Med 2004.

7. Pathak RS PD. A rare differential diagnosis of multiple swellings: Maffucci's syndrome: case report and review of literature Int J Stud Res 2011; 1: 133-7.

8. Hyde GE YCJ, Chu FW. Head and neck manifestations of Maffucci's syndrome: chondrosarcoma of the nasal septum. Am J Otolaryngol. 1995; 16: 272-5.

9. Lewis RJ KA. Maffucci's syndrome: functional and neoplastic significance. Case report and review of the literature. J Bone Joint Surg Am 1973; 55: 1465-79. 


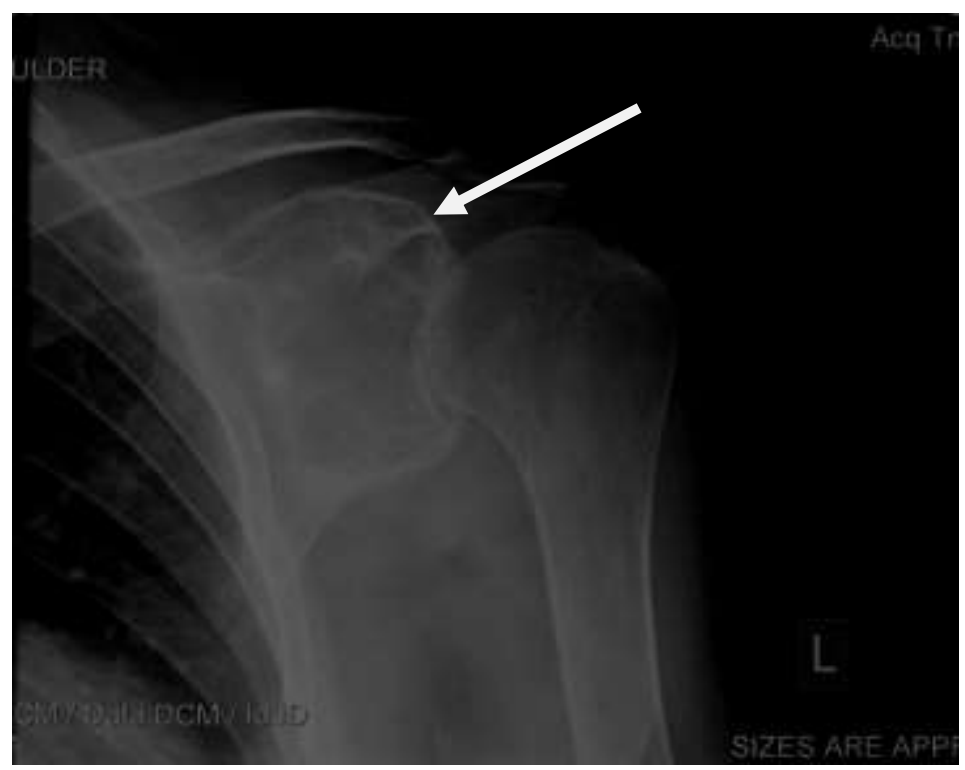

Fig. 1: Large lytic lesion with a chondromatous matrix involving the scapula.

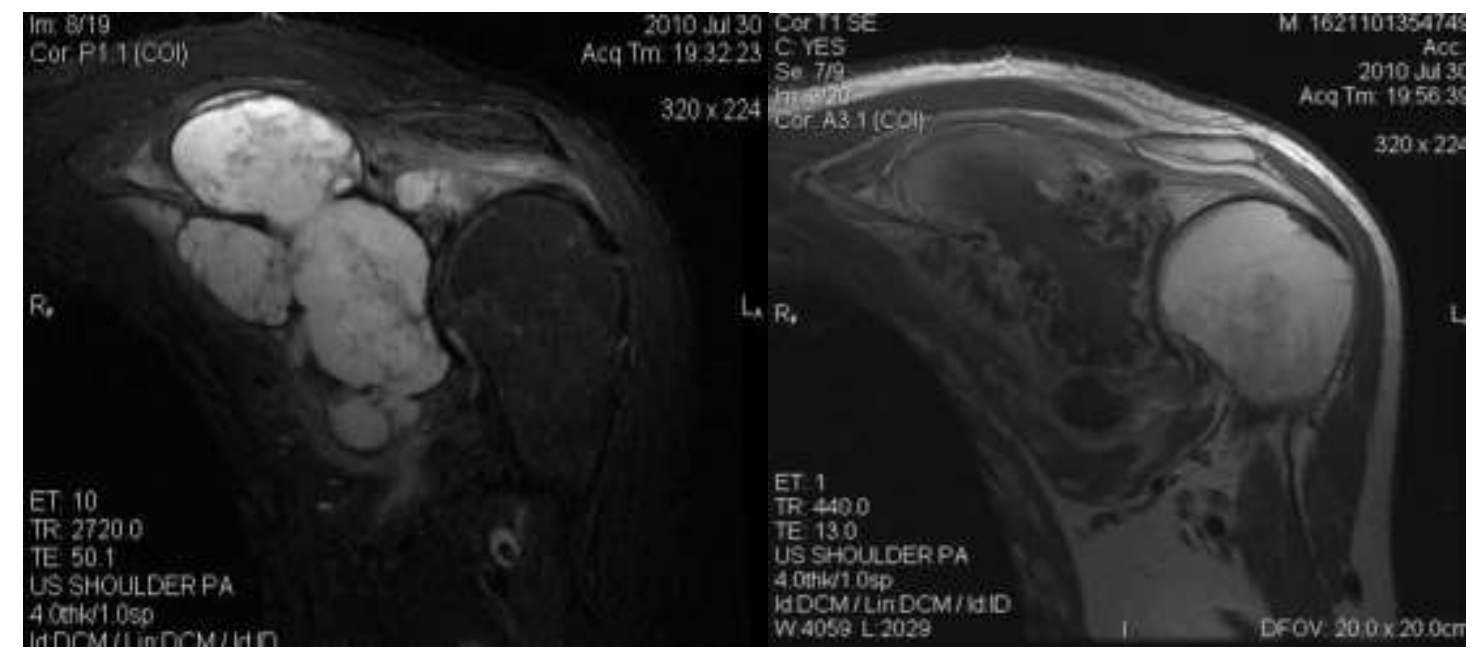

Fig. 2: STIR sequence showing a very high signal in the non-calcified portions of the tumor; $\mathrm{T} 1+\mathrm{Gd}$ shows septal and peripheral rim-like enhancement. 